PROCEEDINGS OF THE

AMERICAN MATHEMATICAL SOCIETY

Volume 136, Number 8, August 2008, Pages 2835-2838

S 0002-9939(08)09383-0

Article electronically published on March 28, 2008

\title{
A NOTE ON MULTIPLIER ALGEBRAS ON REPRODUCING KERNEL HILBERT SPACES
}

\author{
TAVAN T. TRENT
}

(Communicated by Michael T. Lacey)

\begin{abstract}
We construct a simple reproducing kernel space whose multiplier algebra does not satisfy a "corona theorem".
\end{abstract}

Let $\mathcal{H}_{E}$ denote a reproducing kernel Hilbert space on a set $E$. That is, $\mathcal{H}_{E}$ is a Hilbert space of functions on $E$, satisfying:

(i) for every $x \in E$, there exists a $C_{x}<\infty$, so that

$$
|f(x)| \leq C_{x}\|f\|_{\mathcal{H}_{E}}, \text { for all } f \in \mathcal{H}_{E},
$$

and (ii) If $f \in \mathcal{H}_{E}$ and $f(x)=0$ for all $x \in E$, then

$$
\|f\|_{\mathcal{H}_{E}}=0 \text {; i.e. } f=0 \text { in } \mathcal{H}_{E} \text {. }
$$

For $g \in \mathcal{H}_{E}$, define $M_{g}(f)(x)=g(x) f(x)$ for $x \in E$ and $f \in \mathcal{H}_{E}$. Let $\mathcal{M}\left(\mathcal{H}_{E}\right)=\left\{f \in \mathcal{H}_{E}: M_{f} \in \mathcal{B}\left(\mathcal{H}_{E}\right)\right\}$, the multiplier algebra for $\mathcal{H}_{E}$.

When can we solve linear equations in $\mathcal{M}\left(\mathcal{H}_{E}\right)$ ? As a very preliminary step, we might try to characterize when $\mathcal{I}\left(\left\{f_{j}\right\}_{j=1}^{n}\right)=\mathcal{M}\left(\mathcal{H}_{E}\right)$, where $\mathcal{I}\left(\left\{f_{j}\right\}_{j=1}^{n}\right)$ is the ideal generated by $\left\{f_{j}\right\}_{j=1}^{n} \subset \mathcal{M}\left(\mathcal{H}_{E}\right)$. The model characterization is the "corona theorem" of Carleson [1] for $H^{\infty}(D) \quad\left(=\mathcal{M}\left(H^{2}(D)\right)\right)$. Namely, for $\left\{f_{j}\right\}_{j=1}^{n} \subset H^{\infty}(D)$, if $0<\epsilon^{2} \leq \sum_{j=1}^{n}\left|f_{j}(z)\right|^{2}$ for all $z \in D$, then

$$
\mathcal{I}\left(\left\{f_{j}\right\}_{j=1}^{n}\right)=H^{\infty}(D) .
$$

We are interested in whether an abstract "corona theorem" can hold for a general algebra, $\mathcal{M}\left(\mathcal{H}_{E}\right)$. That is, if $\left\{f_{j}\right\}_{j=1}^{n} \subset \mathcal{M}\left(\mathcal{H}_{E}\right)$ and $0<\epsilon^{2} \leq \sum_{j=1}^{n}\left|f_{j}(x)\right|^{2}$ for all $x \in E$, does there exist $\left\{g_{j}\right\}_{j=1}^{n} \subset \mathcal{M}\left(\mathcal{H}_{E}\right)$ with $\sum_{j=1}^{n} f_{j} g_{j}=1$ ?

Of course, by very deep results of Cole (see [2]) and Sibony [3, there are counterexamples to the corona problem of the form $H^{\infty}(\Omega)$ with $\Omega \subset \mathbb{C}^{3}$ and $\Omega \subset \mathbb{C}^{2}$, respectively. Since these algebras can be viewed as multiplier algebras for appropriate Bergman spaces, the answer to the corona question for a general multiplier algebra is "no".

In this note, we give a simple counterexample to the "corona problem" for a multiplier algebra of functions of one variable. This example is strong enough to give that:

Received by the editors February 8, 2007.

2000 Mathematics Subject Classification. Primary 46E22, 47B32.

Key words and phrases. Corona, reproducing kernel.

The author was partially supported by NSF Grant DMS-0400307.

(C)2008 American Mathematical Society Reverts to public domain 28 years from publication 
there is a $g \in \mathcal{M}\left(\mathcal{H}_{E}\right)$, so that $\frac{1}{g} \in \mathcal{H}_{E}$ and $|g(x)| \geq \epsilon>0$ for all $x \in E$, but $\frac{1}{g} \notin \mathcal{M}\left(\mathcal{H}_{E}\right)$.

Clearly, such an example cannot occur in $H^{\infty}(\Omega)$, and, in fact, functions in our space $\mathcal{M}\left(\mathcal{H}_{E}\right)$, will, unfortunately, have no smoothness properties in general.

For $\underline{a}=\left(a_{1}, \ldots, a_{N^{2}}\right) \in l_{N^{2}}^{2}$, let $D_{\underline{a}}$ denote the $N^{2} \times N^{2}$ diagonal matrix, whose diagonal entries are $d_{j j}=a_{j}$ for $j=1, \ldots, N^{2}$. Then our example is based on the following lemma:

Lemma 1. For $N=1,2, \ldots$ there exists an $\underline{a}_{N} \in l_{N^{2}}^{2}$ and an invertible $A_{N} \in$ $M_{N^{2}}(\mathbb{C})$ satisfying:
(i) $\left\|A_{N} D_{\underline{a}_{N}} A_{N}^{-1}\right\|_{B\left(l_{N^{2}}^{2}\right)} \leq 2$
(ii) $\left\|A_{N} D_{\underline{a}_{N}}^{-1} A_{N}^{-1}\right\|_{B\left(l_{N^{2}}^{2}\right)} \geq N$,
(iii) $\left\|A_{N} D_{\underline{a}_{N}}\right\|_{B\left(l_{N^{2}}^{2}\right)} \leq \frac{1}{N^{2}}$,
(iv) $\left\|A_{N} D_{\underline{a}_{N}}^{-1}\right\|_{B\left(l_{N^{2}}^{2}\right)} \leq \frac{1}{N^{2}}$, and
(v) $\left\|D_{\underline{a}_{N}}^{-1}\right\|_{B\left(l_{N^{2}}^{2}\right)} \leq C_{0}$, for a fixed $C_{0}<\infty$ independent of $N$.

Assume that the lemma holds. We proceed to construct our counterexample. Let $\mathcal{H}_{N} \stackrel{\text { def }}{=}\left\{\underline{x} \in \mathbb{C}^{N^{2}}\right.$ : with $\left.\|\underline{x}\|_{\mathcal{H}_{N}} \stackrel{\text { def }}{=}\left\|A_{N}(\underline{x})\right\|_{l^{2}}\right\}$. For each $N=1,2, \ldots$, let $E_{N}=\{\underbrace{\frac{1}{N} e^{\frac{i j t}{2 \pi N^{2}}}}_{z_{N, j}}: j=0, \ldots, N^{2}-1\}$ and $E=\bigcup_{N=1}^{\infty} E_{N} \subset \mathbb{C}$. Note that any pairwise disjoint $E_{N}$ 's with sufficiently many points will work.

For $f: E \rightarrow \mathbb{C}$ and $N=1,2, \ldots$, let $\underline{f}_{N}=\left(f\left(z_{N, 0}\right), \ldots, f\left(z_{N, N^{2}-1}\right)\right)$. Then $\mathcal{H}_{E} \stackrel{\circ}{=}\left\{: E \rightarrow \mathbb{C}:\|f\|_{\mathcal{H}_{E}}^{2} \stackrel{\text { def }}{=} \sum_{N=1}^{\infty}\left\|\underline{f}_{N}\right\|_{\mathcal{H}_{N}}^{2}<\infty\right\}$. Since $\mathcal{H}_{E}$ is isomorphic to $\bigoplus_{N=1}^{\infty} \mathcal{H}_{N}$, we see that $\mathcal{H}_{E}$ is a Hilbert space of functions on $E$. Also, for $z_{N, j} \in E$,

$$
\begin{aligned}
\left|f\left(z_{N, j}\right)\right| \leq\left\|\underline{f}_{N}\right\|_{l_{N^{2}}^{2}} & \leq\left\|A_{N}^{-1}\right\|_{B\left(l_{N^{2}}^{2}\right)}\left\|A_{N}\left(\underline{f}_{N}\right)\right\|_{l_{N^{2}}^{2}} \\
& =\left\|A_{N}^{-1}\right\|_{B\left(l_{N^{2}}^{2}\right)}\left\|\underline{f}_{N}\right\|_{\mathcal{H}_{N}} \\
& \leq\left\|A_{N}^{-1}\right\|_{B\left(l_{N^{2}}^{2}\right)}\|f\|_{\mathcal{H}_{E}}
\end{aligned}
$$

Clearly, if $f: E \rightarrow \mathbb{C}$ vanishes on $E$, then $\|f\|_{\mathcal{H}_{E}}=0$. Thus $\mathcal{H}_{E}$ is a reproducing kernel Hilbert space on $E$. 
Suppose that $f \in \mathcal{M}\left(\mathcal{H}_{E}\right)$; then a computation shows that, for $h \in \mathcal{H}_{E}$,

$$
\begin{aligned}
\left\|M_{f}(h)\right\|_{\mathcal{H}_{E}}^{2} & =\sum_{N=1}^{\infty}\left\|A_{N}\left((\underline{f h})_{N}\right)\right\|_{l_{N^{2}}^{2}}^{2} \\
& =\sum_{N=1}^{\infty}\left\|A_{N}\left(D_{\underline{f}_{N}}(\underline{h})_{N}\right)\right\|_{l_{N^{2}}^{2}}^{2} \\
& =\sum_{N=1}^{\infty}\left\|\left(A_{N} D_{\underline{f}_{N}} A_{N}^{-1}\right)\left(A_{N}\left(\underline{h}_{N}\right)\right)\right\|_{l_{N^{2}}^{2}}^{2} .
\end{aligned}
$$

From this equality it follows that

$$
\left\|M_{f}\right\|_{B\left(\mathcal{H}_{E}\right)}=\sup _{N \in \mathbb{N}}\left\{\left\|A_{N} D_{\underline{f}_{N}} A_{N}^{-1}\right\|_{B\left(l_{N^{2}}^{2}\right)}\right\} .
$$

Using the lemma to get the appropriate sequence of $\underline{a}_{N}$ 's, we define $g: E \rightarrow \mathbb{C}$, so that $\underline{g}_{N}=\underline{a}_{N}$, for $N=1,2, \ldots$. Then $g \in \mathcal{H}_{E}$, since by (iii) of the lemma

$$
\begin{aligned}
\|g\|_{\mathcal{H}_{E}}^{2} & =\sum_{N=1}^{\infty}\left\|\underline{g}_{N}\right\|_{\mathcal{H}_{N}}^{2}=\sum_{N=1}^{\infty}\left\|A_{N}\left(\underline{a}_{N}\right)\right\|_{l_{N^{2}}^{2}}^{2} \\
& =\sum_{N=1}^{\infty}\left\|A_{N} D_{\underline{a}_{N}}\left(\underline{u}_{N}\right)\right\|_{l_{N^{2}}^{2}}^{2} \leq \sum_{N=1}^{\infty} \frac{N^{2}}{N^{4}}<\infty
\end{aligned}
$$

where $\underline{u}_{N}$ denotes the vector in $\mathbb{C}^{N^{2}}$ consisting of all 1's. A similar argument, using (iv), shows that $\frac{1}{g} \in \mathcal{H}_{E}$.

From (v), for all $N=1,2, \ldots$ and $j=0, \ldots, N^{2}-1$, we have $\left|g\left(z_{N, j}\right)\right| \geq \frac{1}{C_{0}}$. So $|g(x)| \geq \frac{1}{C_{0}}$, for all $x \in E$.

By our construction,

$$
\left\|M_{g}\right\|_{B\left(\mathcal{H}_{E}\right)}=\sup _{N \in \mathbb{N}}\left\{\left\|A_{N} D_{\underline{g}_{N}} A_{N}^{-1}\right\|_{B\left(l_{N^{2}}^{2}\right.}\right\} \leq 2 \text {, by (i). }
$$

By a similar estimate, using (ii) we get that

$$
\left\|M_{\frac{1}{g}}\right\|_{B\left(\mathcal{H}_{E}\right)}=\sup _{N \in \mathbb{N}}\left\{\left\|A_{N} D_{\underline{g}_{N}}^{-1} A_{N}^{-1}\right\|_{B\left(l_{N^{2}}^{2}\right)}\right\}=\infty .
$$

This completes the construction.

We finish with a proof of the lemma.

Proof. For $N=1,2, \ldots$ construct the $N^{2} \times N^{2}$ matrix

$$
B_{N}=\left[\begin{array}{cccccc}
\frac{1}{1+\frac{1}{N}+\frac{1}{N^{3}}} & -1 & 0 & 0 & \cdots & 0 \\
0 & \frac{1}{1+\frac{1}{N}+\frac{2}{N^{3}}} & -1 & 0 & \cdots & 0 \\
0 & 0 & -\frac{1}{1+\frac{1}{N}+\frac{3}{N^{3}}} 1 & -1 & \cdots & 0 \\
0 & 0 & 0 & \ddots & \ddots & \vdots \\
\vdots & \vdots & \vdots & \ddots & \ddots & -1 \\
0 & 0 & 0 & \cdots & 0 & \frac{1}{1+\frac{1}{N}+\frac{N^{2}}{N^{3}}}
\end{array}\right] .
$$

Clearly, $\sigma\left(B_{N}\right)=\left\{\frac{1}{1+\frac{1}{N}+\frac{k}{N^{3}}}: 1 \leq k \leq N^{2}\right\} \subset D$, the open unit disk. 
Since the spectral radius of $B_{N}$ is strictly less than 1 , there exists a $T_{N} \in$ $M_{N^{2}}(\mathbb{C})$ so that

$$
\left\|T_{N}^{-1} B_{N} T_{N}\right\|_{B\left(l_{N^{2}}^{2}\right)} \leq 1
$$

Now $\left\|B_{N}^{-1}\right\|_{B\left(l_{N^{2}}^{2}\right)} \geq \prod_{k=1}^{N^{2}}\left(1+\frac{1}{N}+\frac{k}{N^{3}}\right) \geq N \quad$ and $\quad\left\|B_{N}\right\|_{B\left(l_{N^{2}}^{2}\right)} \leq 2 . \quad$ Set $C_{N}=T_{N}^{-1} B_{N} T_{N}$. Then

$$
\begin{aligned}
\left\|C_{N}\right\|_{B\left(l_{N^{2}}^{2}\right)} & \leq 1, \\
\left\|T_{N} C_{N} T_{N}^{-1}\right\|_{B\left(l_{N^{2}}^{2}\right)} & \leq 2, \\
\text { and }\left\|T_{N} C_{N}^{-1} T_{N}^{-1}\right\|_{B\left(l_{N^{2}}^{2}\right)} & \geq N .
\end{aligned}
$$

Since $\sigma\left(C_{N}\right)=\sigma\left(B_{N}\right), \quad \sigma\left(C_{N}\right)$ consists of the $N^{2}$ distinct eigenvalues $\left\{\frac{1}{1+\frac{1}{N}+\frac{k}{N^{3}}}: 1 \leq k \leq N^{2}\right\}$. If we denote by $D_{N}$ the $N^{2} \times N^{2}$ matrix with the same diagonal as $B_{N}$, then $C_{N}$ is similar to $D_{N}$, say $C_{N}=P_{N} D_{N} P_{N}^{-1}$. Set $\quad A_{N}=\frac{T_{N} P_{N}}{N^{2}\left\|T_{N} P_{N}\right\|_{B\left(l_{N^{2}}^{2}\right)}\left(\left\|D_{N}\right\|_{B\left(l^{2}\right)^{2}}+\left\|D_{N}^{-1}\right\|_{B\left(l_{N}^{2}\right)}^{2}\right)}$. Then the lemma follows with $\underline{a}_{N}=\left(1+\frac{1}{N}+\frac{1}{N^{3}}, 1+\frac{1}{N}+\frac{2}{N^{3}}, \ldots, 1+\frac{1}{N}+\frac{N^{2}}{N^{3}}\right) \in \mathbb{C}^{N^{2}}$.

\section{REFERENCES}

[1] L. Carleson, Interpolation by bounded analytic functions and the corona problem, Annals of Math. 76 (1962), 547-559. MR0141789 (25:5186)

[2] T. W. Gamelin, Uniform Algebras and Jensen Measures, London Mathematical Society Lecture Note Series 32, Cambridge University Press, Cambridge, 1978. MR521440 (81a:46058)

[3] N. Sibony, Un exemple de domain pseudoconvexe régulier où l'équation $\bar{\partial} u=f$ n'admet pas de solution bornée pour $f$ bornée, Invent. Math. 62 (1980/81), 235-242. MR595587 (82c:32020)

Department of Mathematics, The University of Alabama, Box 870350, Tuscaloosa, AlaBAma 35487-0350

E-mail address: ttrent@as.ua.edu 